\title{
Align announces new iTero updates and connectivity
}

Align Technology has announced multiple updates associated with the iTero Element family of Intraoral scanners (Element, Element 2 and Element Flex) which includes a new software upgrade with enhanced functionality and connectivity with important digital dentistry leader: exocad.

With these updates, iTero has strengthened its position in restorative and dental practice workflow and is able to help dentists offer a better, faster patient experience.

Thanks to the 1.7 iTero scanner software upgrade, which will be available through a gradual release starting in late November, general dentists and orthodontists will have access to new features which improve scan quality and provide additional functionality for patient data protection. These improved features include:

- Improved image quality and resolution of restorative model

- Direct visualisation of high resolution scan in 'scan mode'

- Improved scan process for prep segments

- Inactivity log out feature that activates after a pre-defined period of system inactivity for greater privacy of patient information.

In addition, a separate iTero software update also provided customers with an improvement in their experience at myitero.com, with clear visibility of file download status.

Align has also announced that iTero Intraoral scanner open chair-side milling workflow with exocad ChairsideCAD software offers a fully-validated workflow between iTero Element Intraoral scanner and exocad ${ }^{\circ}$ ChairsideCAD to enable in-house milling of dental restoration on any compatible milling machine.

With the flexibility of being able to choose chairside workflow that works for their practice, dentists can focus on offering a better patient experience and faster treatment through same-day dentistry.

Practice owner and cosmetic dentist of The Courtyard Clinical Academy, Marcos White said of iTero: 'It has proved game changing and transformational to our case acceptance and reputation. An iTero will give you return on investment at the consultation stage'.

White will present a webinar on 24 January 2019 at 7 pm entitled Multi disciplinary intra oral scanning. From digital consultation to full arch implants. To pre-register, please email catherinedomanski@ positivecomm.com.

The latest news on the iTero Element scanner portfolio is available by visiting www.itero.com or www.facebook.com/ iterodigitalimpressionsystem/.

Align Technology has announced multiple updates associated with the iTero Element family of Intraoral scanners (Element, Element 2 and Element Flex) which includes a new software upgrade with enhanced functionality and connectivity with important digital dentistry leader: exocad.

\section{PRP - putting the record straight}

The subject of platelet-rich plasma, also known as PRP can be confusing.

Over the years, since Drs Arun Garg and Robert Marx first placed patients' own blood in a centrifuge to bring plasma to the top, fibrin to the middle and erythrocytes to the bottom, commercial forces and a lack of industry standardisation has led to myriad nomenclatures, including, but not limited to PRF, i-PRF, A-PRF etc. However, they are all the same product.

Garg is an internationally-recognised dental educator and surgeon who, for more than 20 years, served as a full-time professor of surgery in the division of oral and maxillofacial surgery and as director of residency training at the University of Miami, Leonard M. Miller School of Medicine.

$\mathrm{He}$ is considered the world's pre-eminent authority on bone biology, bone harvesting and bone grafting for dental implant surgery. Alongside Dr Marx, he pioneered the use of platelet-rich plasma (PRP).

Looking to set the record straight on PRP and speaking about its evolution, Garg said: 'I could play the same game and introduce 5th generation PRP but that would be disingenuous.

'The truth is, PRP is PRP whatever way you look at it, and PRF, i-PRF and so on are all PRP too! The only difference between the 1990s when Robert and I created this by-product of patients' own blood and now is that we have kept up with changing technology.

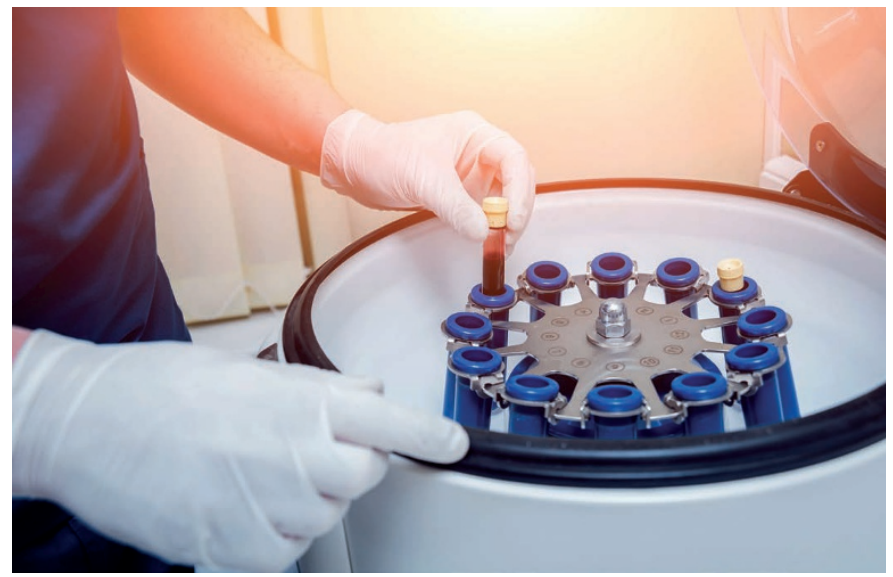

All autologous blood concentrates are the same, irrespective of what another user may call it.'

Teaming up with Garg to make sense of the confusion is the prestigious publisher Quintessence, hosting his blog on autologous blood concentrates on its website.

If you would like to know more about how PRP misconceptions have developed over the years, to learn the truth behind the abbreviations and to buy Garg's book on the topic, please visit http://www. quintpub.net/news/2018/08/autologous-blood-concentrates-makingsense-of-all-the-hype/\#.W9a-6zGYSUn. 